\title{
Generalized parton distributions and pseudodistributions
}

\author{
A. V. Radyushkin $\odot$ \\ Old Dominion University, Norfolk, Virginia 23529, USA \\ and Thomas Jefferson National Accelerator Facility, Newport News, Virginia 23606, USA
}

(Received 1 October 2019; published 16 December 2019)

\begin{abstract}
We derive one-loop matching relations for the Ioffe-time distributions (ITDs) related to the pion distribution amplitude (DA) and generalized parton distributions (GPDs). They are obtained from a universal expression for the one-loop correction in an operator form, and will be used in the ongoing lattice calculations of the pion DA and GPDs within the parton pseudodistributions approach.
\end{abstract}

DOI: 10.1103/PhysRevD.100.116011

\section{INTRODUCTION}

Extraction of parton distribution functions (PDFs) from lattice simulations attracts now a considerable interest and efforts (for a recent review see Ref. [1]). An intensive development in this field has started with the paper by X. Ji [2], who proposed the concept of parton quasi-distributions (quasi-PDFs) formalized later within a general framework of the large momentum effective theory (LaMET) [3]. The basic idea of Ref. [2] (preceded in Refs. [4,5]) to study equal-time correlators is also used in the "good lattice cross sections" approach [6,7] and in the pseudo-PDF approach [8-10].

The conversion of the Euclidean-space lattice data into the standard light-cone PDFs is performed with the help of the matching relations. In the quasi-PDF approach, such relations were derived for the usual PDFs [2,11-15], the pion distribution amplitude (DA) [16] and generalized parton distributions (GPDs) [16-18]. Matching relations between pseudo-PDFs and the usual light-cone PDFs were discussed in Refs. [13,19-22].

It should be noted that, in all the papers mentioned above, the derivation of the matching relations was based on separate calculations of the relevant one-loop Feynman diagrams. However, as pointed out in our paper [20], the one-loop correction in the coordinate-representation approach of Ref. [23] may be calculated in the operator form, i.e., without specifying the matrix element characteristic of a particular parton distribution.

The diagram by diagram results of such one-loop calculation for a nonsinglet quark operator are given in Ref. [20], and they were used there to obtain the matching

Published by the American Physical Society under the terms of the Creative Commons Attribution 4.0 International license. Further distribution of this work must maintain attribution to the author(s) and the published article's title, journal citation, and DOI. Funded by SCOAP . relations between the nonsinglet pseudo-PDF and the corresponding PDF. It was also stated there that the same result obtained on the operator level may be used to derive matching relations for the pion distribution amplitude and the nonsinglet generalized parton distributions.

It is the goal of the present paper to describe the derivation of these matching relations. They can be used in future lattice extractions of the pion DA and nonsinglet GPDs within the pseudo-PDF approach.

To make the paper self-contained, we describe in Sec. II the derivation of the known matching relations for nonsinglet PDFs. In Sec. III, we derive matching relations for the pion distribution amplitude. The matching relations for nonsinglet GPDs are derived in Sec. IV. Section V contains the summary of the paper.

\section{MATCHING CONDITIONS IN THE COORDINATE SPACE}

\section{A. Operators and parton distributions}

In the present paper, we will consider the valence parton distribution functions, the pion distribution amplitude (DA) and nonsinglet generalized parton distributions. They all are given by matrix elements of nonsinglet operators of a generic form

$$
\mathcal{O}^{\alpha}(z) \equiv \bar{\psi}(0) \Gamma^{\alpha} \hat{E}(0, z ; A) \psi(z),
$$

where $\Gamma^{\alpha}=\gamma^{\alpha}$ or $\gamma^{\alpha} \gamma_{5}$. The factor $\hat{E}(0, z ; A)$ is the standard $0 \rightarrow z$ straight-line gauge link in the quark (fundamental) representation

$$
\hat{E}(0, z ; A) \equiv P \exp \left[i g z_{\nu} \int_{0}^{1} d t \hat{A}^{\nu}(t z)\right] .
$$

In particular, studying the parton distribution functions, we deal with the forward matrix elements 


$$
M^{\alpha}(z, p) \equiv\left\langle p\left|\mathcal{O}^{\alpha}(z)\right| p\right\rangle
$$

between the hadronic states $|p\rangle$ with momentum $p$. By Lorentz invariance, $M^{\alpha}(z, p)$ may be represented as a sum of two structures

$$
M^{\alpha}(z, p)=2 p^{\alpha} \mathcal{M}\left(-(z p),-z^{2}\right)+z^{\alpha} \mathcal{M}_{z}\left(-(z p),-z^{2}\right)
$$

involving the amplitudes depending on two Lorentz scalars: the interval $z^{2}$ and the invariant $(p z) \equiv-\nu$, the Ioffe time [24].

The twist-2 PDF is determined by the Ioffe-time pseudodistribution $\mathcal{M}\left(\nu,-z^{2}\right)$, while $\mathcal{M}_{z}\left(\nu,-z^{2}\right)$ is a purely higher-twist contamination. It can be eliminated by an appropriate choice of $z$ and $p$. The usual way to define twist-2 PDF is to use $z$ in a purely "minus" light-cone direction, i.e., $z=z_{-}$and take $\alpha=+$. To exclude $\mathcal{M}_{z}$ in lattice calculations, one may use $z=z_{3}$ and $\alpha=0$, as suggested in Ref. [25]. We will follow this prescription for all the parton distributions that we consider in the present paper.

\section{B. One-loop correction in the operator form}

The one-loop correction to $\mathcal{O}^{0}\left(z_{3}\right)$ was calculated in the operator form in Ref. [20], and is given by

$$
\begin{aligned}
\delta \mathcal{O}^{0}\left(z_{3}\right)= & -\frac{\alpha_{s}}{2 \pi} C_{F} \int_{0}^{1} d u \int_{0}^{1-u} d v \bar{\psi}\left(u z_{3}\right) \Gamma^{0} \psi\left(\bar{v} z_{3}\right) \\
& \times\left\{\left(\delta(v)\left[\frac{\bar{u}}{u}\right]_{+}+\delta(u)\left[\frac{\bar{v}}{v}\right]_{+}+1\right) \ln \left[z_{3}^{2} \mu_{\mathrm{IR}}^{2} \frac{e^{2 \gamma_{E}+1}}{4}\right]\right. \\
& +2\left(\delta(v)\left[\frac{\ln u}{u}\right]_{+}+\delta(u)\left[\frac{\ln v}{v}\right]_{+}-1\right) \\
& \left.+Z\left(z_{3}\right) \delta(u) \delta(v)\right\}
\end{aligned}
$$

Here we use the notation $\bar{v}=1-v, \bar{u}=1-u$, etc. In what follows, we will also use the variable $w=1-u-v$. The plus-prescription at zero is defined by

$$
\int_{0}^{1} d u\left[\frac{\bar{u}}{u}\right]_{+} F(u)=\int_{0}^{1} d u \frac{\bar{u}}{u}[F(u)-F[0]],
$$

assuming that $F(0)$ is finite.

In our result (2.5), we have used the dimensional regularization for collinear singularities, and applied the $\overline{\mathrm{MS}}$ scheme subtraction with $\mu_{\mathrm{IR}}$ serving as the scale parameter.

The function $Z(z)$ accumulates information about local corrections associated with the ultraviolet-divergent contributions. This function is also known (see Ref. [13]), but, in the pseudo-PDF approach, we do not need its explicit form. As we will see, such terms cancel when one forms the reduced Ioffe-time pseudodistributions.

In Feynman gauge, the terms

$$
\left(\delta(v)\left[\frac{\bar{u}}{u}\right]_{+}+\delta(u)\left[\frac{\bar{v}}{\bar{v}}\right]_{+}\right)
$$

and

$$
\left(\delta(v)\left[\frac{\ln u}{u}\right]_{+}+\delta(u)\left[\frac{\ln v}{v}\right]_{+}\right)
$$

containing $\delta(u)$ or $\delta(v)$ in the coefficient function of Eq. (2.5) are produced by vertex diagrams, while the " +1 " and " $-1 " u, v$-independent additions to them come from the box diagram (see Ref. [20]). So, we will use sometimes "vertex" and "box" to refer to these two types of contributions.

\section{Matching for parton distribution functions}

In the PDF case, the one-loop correction to $M^{0}\left(z_{3}, p\right)$ is given by the forward matrix element $\left\langle p\left|\delta \mathcal{O}^{0}\left(z_{3}\right)\right| p\right\rangle$. The right-hand side of Eq. (2.5) brings then the matrix element

$$
\mathcal{M}_{0}(u \nu, \bar{v} \nu)=\left\langle p\left|\bar{\psi}\left(u z_{3}\right) \Gamma^{0} \psi\left(\bar{v} z_{3}\right)\right| p\right\rangle,
$$

where $\nu=p_{3} z_{3}$ is the Ioffe time [24].

Using translation invariance, the "vertex" terms containing $\delta(u)$ or $\delta(v)$ are trivially reduced to onedimensional integrals involving, say, $(\bar{u} / u)_{+} \mathcal{M}_{0}(0, \bar{u} \nu)$ or $(\bar{v} / v)_{+} \mathcal{M}_{0}(0, \bar{v} \nu)$. Changing $u$ or $v$ to a common variable $1-w$, we get the $w$-integral of $2(w / \bar{w})_{+} \mathcal{M}_{0}(0, w \nu)$ with the plus-prescription at $w=1$.

For the "box" terms, we get

$$
\begin{gathered}
\int_{0}^{1} d u \int_{0}^{1-u} d v \mathcal{M}_{0}(0,(1-u-v) \nu) \\
=\int_{0}^{1} d w(1-w) \mathcal{M}_{0}(0, w \nu) .
\end{gathered}
$$

We can represent $(1-w)$ as the sum of the term $(1-w)_{+}$ that has the plus-prescription at $w=1$ and the deltafunction term $\frac{1}{2} \delta(\bar{w})$ that we add to $Z\left(z_{3}\right)$, denoting the changed $Z$-function by $\tilde{Z}\left(z_{3}\right)$. As a result, we have

$$
\begin{aligned}
\mathcal{M}\left(\nu, z_{3}^{2}\right)= & {\left[1-\frac{\alpha_{s}}{2 \pi} C_{F} \tilde{Z}\left(z_{3}\right)\right] \mathcal{M}_{0}(\nu) } \\
& -\frac{\alpha_{s}}{2 \pi} C_{F} \int_{0}^{1} d w\left\{\frac{1+w^{2}}{1-w} \ln \left(z_{3}^{2} \mu_{\mathrm{IR}}^{2} \frac{e^{2 \gamma_{E}+1}}{4}\right)\right. \\
& \left.+4 \frac{\ln (1-w)}{1-w}-2(1-w)\right\}_{+} \mathcal{M}_{0}(w \nu),
\end{aligned}
$$


where we have abbreviated $\mathcal{M}_{0}(0, \nu)$ to $\mathcal{M}_{0}(\nu)$ and similarly for $\mathcal{M}_{0}(0, w \nu)$.

The structure of Eq. (2.5) implies a scenario in which the $z_{3}^{2}$-dependence at short distances is determined by the "hard" $\operatorname{logarithms} \ln z_{3}^{2}$ generated from the initially "soft" distribution $\mathcal{M}_{0}\left(\nu, z_{3}^{2}\right)$ having only a polynomial dependence on $z_{3}^{2}$ that is negligible for small $z_{3}^{2}$. For this reason, we have skipped the $z_{3}^{2}$-dependence in the argument of $\mathcal{M}_{0}$-functions, leaving just their $\nu$-dependence. The combination

$$
B(w)=\left[\frac{1+w^{2}}{1-w}\right]_{+}
$$

is the nonsinglet Altarelli-Parisi (AP) evolution kernel [26]. The latter is usually defined for PDFs, i.e., in the momentum-fraction space. However, introducing the pseudo-PDF $\mathcal{P}\left(x, z_{3}^{2}\right)[8]$ by

$$
\mathcal{P}\left(x, z_{3}^{2}\right)=\frac{1}{2 \pi} \int_{-\infty}^{\infty} d \nu e^{-i x \nu} \mathcal{M}\left(\nu, z_{3}^{2}\right)
$$

or by the inverse transformation

$$
\mathcal{M}\left(\nu, z_{3}^{2}\right)=\int_{-1}^{1} d y e^{i y \nu} \mathcal{P}\left(y, z_{3}^{2}\right),
$$

with $\mathcal{P}(x, 0) \equiv f(x)$ being the usual PDF, we see that Eq. (2.9) converts into

$$
\begin{aligned}
\mathcal{P}\left(x, z_{3}^{2}\right)= & f(x)-\frac{\alpha_{s}}{2 \pi} C_{F} \ln \left(z_{3}^{2}\right) \int_{0}^{1} d w B(w) \\
& \times \int_{-1}^{1} d y \delta(x-w y) f(y)+\cdots \\
= & f(x)-\frac{\alpha_{s}}{2 \pi} C_{F} \ln \left(z_{3}^{2}\right) \int_{-1}^{1} \frac{d y}{|y|} B(x / y) f(y)+\cdots,
\end{aligned}
$$

in which the $\ln z_{3}^{2}$ part has the standard form of the DGLAP (for Dokshitzer-Gribov-Lipatov-Altarelli-Parisi [26-28]) evolution equation.

The next step is to introduce the reduced Ioffe-time pseudodistribution

$$
\mathfrak{M}\left(\nu, z_{3}^{2}\right) \equiv \frac{\mathcal{M}\left(\nu, z_{3}^{2}\right)}{\mathcal{M}\left(0, z_{3}^{2}\right)}
$$

of Refs. [8-10]. When the momentum $p$ is also oriented in the $z_{3}$ direction, i.e., $p=\left\{E, 0_{\perp}, p_{3}\right\}$, the function $\mathcal{M}\left(0, z_{3}^{2}\right)$ corresponds to the "rest-frame" $p_{3}=0$ distribution. According to Eq. (2.9), it is given by

$$
\mathcal{M}\left(0, z_{3}^{2}\right)=\mathcal{M}_{0}(0)\left[1-\frac{\alpha_{s}}{2 \pi} C_{F} \tilde{Z}\left(z_{3}\right)\right]
$$

As a result, the $\tilde{Z}\left(z_{3}\right)$ terms disappear from the $\mathcal{O}\left(\alpha_{s}\right)$ correction to the ratio $\mathcal{M}\left(\nu, z_{3}^{2}\right) / \mathcal{M}\left(0, z_{3}^{2}\right)$, and we have

$$
\begin{aligned}
\mathfrak{M}\left(\nu, z_{3}^{2}\right) \\
=\mathfrak{M}_{0}(\nu)-\frac{\alpha_{s}}{2 \pi} C_{F} \int_{0}^{1} d w\left[\frac{1+w^{2}}{1-w} \ln \left(z_{3}^{2} \mu_{\mathrm{IR}}^{2} \frac{e^{2 \gamma_{E}+1}}{4}\right)\right. \\
\left.\quad+4 \frac{\ln (1-w)}{1-w}-2(1-w)\right]_{+} \mathfrak{M}_{0}(w \nu) .
\end{aligned}
$$

Such a cancellation of ultraviolet terms for $\mathfrak{M}\left(\nu, z_{3}^{2}\right)$ will persist in higher $\alpha_{s}$ orders, reflecting the multiplicative renormalizability of the ultraviolet divergences of $\mathcal{M}\left(\nu, z_{3}^{2}\right)$ [29-31].

A similar calculation can be performed for the light-cone Ioffe-time distribution $\mathcal{I}\left(\nu, \mu^{2}\right)$ [32] obtained by taking $z^{2}=0$ in $\mathfrak{M}\left(\nu,-z^{2}\right)$ and regularizing the resulting lightcone singularities using dimensional regularization and the $\overline{\mathrm{MS}}$ subtraction specified by a factorization scale $\mu$. The result may be symbolically written as

$$
\begin{aligned}
\mathcal{I}\left(\nu, \mu^{2}\right)= & \mathfrak{M}_{0}(\nu)-\frac{\alpha_{s}}{2 \pi} C_{F} \int_{0}^{1} d w\left[\frac{1+w^{2}}{1-w}\right]_{+} \\
& \times \ln \left(\mu_{\mathrm{IR}}^{2} / \mu^{2}\right) \mathfrak{M}_{0}(w \nu) .
\end{aligned}
$$

Combining Eqs. (2.16) and (2.17) gives the matching condition for the light-cone ITD [13,19-22]

$$
\begin{aligned}
\mathcal{I}\left(\nu, \mu^{2}\right)= & \mathfrak{M}\left(\nu, z_{3}^{2}\right)+\frac{\alpha_{s}}{2 \pi} C_{F} \int_{0}^{1} d w \mathfrak{M}\left(w \nu, z_{3}^{2}\right) \\
& \times\left[\frac{1+w^{2}}{1-w} \ln \left(z_{3}^{2} \mu^{2} \frac{e^{2 \gamma_{E}+1}}{4}\right)\right. \\
& \left.+4 \frac{\ln (1-w)}{1-w}-2(1-w)\right]_{+}
\end{aligned}
$$

that allows to get $\mathcal{I}\left(\nu, \mu^{2}\right)$ from lattice data on $\mathfrak{M}\left(\nu, z_{3}^{2}\right)$. By definition [32], the light-cone ITD $\mathcal{I}\left(\nu, \mu^{2}\right)$ is related to the PDF $f\left(x, \mu^{2}\right)$ by

$$
\mathcal{I}\left(\nu, \mu^{2}\right)=\int_{-1}^{1} d x e^{i x \nu} f\left(x, \mu^{2}\right)
$$

Thus, $f\left(x, \mu^{2}\right)$ is formally given by the inverse transformation

$$
f\left(x, \mu^{2}\right)=\frac{1}{2 \pi} \int_{-\infty}^{\infty} d \nu e^{-i x \nu} \mathcal{I}\left(\nu, \mu^{2}\right) .
$$

However, lattice calculations provide $\mathcal{I}\left(\nu, \mu^{2}\right)$ in a rather limited range of $\nu$, which makes taking this Fourier 
transform rather tricky (see Ref. [33] for a detailed discussion). An easier way was proposed in our paper [8]. The idea is to assume some parametrization for $f\left(x, \mu^{2}\right)$ similar to those used in global fits (see, e.g., Ref. [34]), and to incorporate Eq. (2.19) to fit its parameters using the lattice data for $\mathcal{I}\left(\nu, \mu^{2}\right)$.

An equivalent realization of this idea (similar to that of Ref. [35]) is to represent $\mathfrak{M}\left(\nu, z_{3}^{2}\right)$ in terms of $\mathcal{I}\left(\nu, \mu^{2}\right)$ (see, e.g., Eq. (5.1) in Ref. [36]), which, in turn is written through its definition (2.19) as a Fourier transform of PDF

$$
\begin{aligned}
\mathfrak{M}\left(\nu, z_{3}^{2}\right)= & \int_{-1}^{1} d x e^{i x \nu} f\left(x, \mu^{2}\right)-\frac{\alpha_{s}}{2 \pi} C_{F} \int_{-1}^{1} d x f\left(x, \mu^{2}\right) \\
& \times \int_{0}^{1} d w\left(e^{i x w \nu}-e^{i x \nu}\right)\left[\frac{1+w^{2}}{1-w} \ln \left(z_{3}^{2} \mu^{2} \frac{e^{2 \gamma_{E}+1}}{4}\right)\right. \\
& \left.+4 \frac{\ln (1-w)}{1-w}-2(1-w)\right] \\
\equiv & \int_{-1}^{1} d x\left[e^{i x \nu}-\frac{\alpha_{s}}{2 \pi} C_{F} R\left(x \nu, z_{3}^{2} \mu^{2}\right)\right] f\left(x, \mu^{2}\right) .
\end{aligned}
$$

The kernel $R\left(x \nu, z_{3}^{2} \mu^{2}\right)$, introduced in the equation above, may be calculated as a closed-form expression and is given by

$$
\begin{aligned}
& R\left(y, z_{3}^{2} \mu^{2}\right) \\
& =\frac{1}{y^{2}} \ln \left(z_{3}^{2} \mu^{2} \frac{e^{2 \gamma_{E}+1}}{4}\right)\left\{1-i y+e^{i y}\right. \\
& \left.\quad \times\left[2 i y-1+2 y^{2}\left\{\operatorname{Ci}(y)-i \operatorname{Si}(y)-\ln (y)-\gamma_{E}+\frac{3}{4}\right\}\right]\right\} \\
& \quad+4 i y e^{i y}{ }_{3} F_{3}(1,1,1 ; 2,2,2 ;-i y) \\
& \quad-\frac{2}{y^{2}}\left[1+i y-e^{i y}\left(1+y^{2} / 2\right)\right]
\end{aligned}
$$

(see also Ref. [13]), where $\operatorname{Ci}(y)$ and $\operatorname{Si}(y)$ are the integral cosine and sine functions, and ${ }_{3} F_{3}(1,1,1$; $2,2,2 ;-i y)$ is a hypergeometric function.

One may split $f(x)$ in its symmetric $f^{+}(x)$ and antisymmetric $f^{-}(x)$ parts. For positive $x$, they are related to the quark $f_{q}(x)$ and antiquark $f_{\bar{q}}(x)$ distributions by $f^{+}(x)=f_{q}(x)-f_{\bar{q}}(x)$ and $f^{-}(x)=f_{q}(x)+f_{\bar{q}}(x)$, respectively (see, e.g., Ref. [10]). Then the real part of $R\left(y, z_{3}^{2} \mu^{2}\right)$ generates the real part of $\mathfrak{M}\left(\nu, z_{3}^{2}\right)$ from $f^{+}(x)$, while the imaginary part of $R\left(y, z_{3}^{2} \mu^{2}\right)$ connects the imaginary part of $\mathfrak{M}\left(\nu, z_{3}^{2}\right)$ with $f^{-}(x)$.

Thus, assuming some parametrizations for the $f^{ \pm}\left(x, \mu^{2}\right)$ distributions, one can fit their parameters and $\alpha_{s}$ using Eqs. (2.21), (2.22) and the lattice data for $\mathfrak{M}\left(\nu, z_{3}^{2}\right)$.

\section{MATCHING FOR PION DISTRIBUTION AMPLITUDE}

\section{A. Definition and general properties}

The pion distribution amplitude, initially introduced in our 1977 paper (see Ref. [37]) may be defined using the matrix element

$$
M^{\alpha}(z, p)=\left\langle 0\left|\bar{\psi}(0) \gamma^{\alpha} \gamma_{5} \hat{E}(0, z ; A) \psi(z)\right| p\right\rangle
$$

where $|p\rangle$ is a pion state with momentum $p$. A similar object was introduced within the light-front quantization formalism [38] (see Ref. [39] for comparison of the two definitions).

Again, on the lattice, we take $z=z_{3}$ and $\alpha=0$ to extract the $p^{\alpha} \mathcal{M}\left(\nu,-z^{2}\right)$ part of the decomposition of $M^{\alpha}(z, p)$ over Lorentz structures, and then form the reduced Ioffetime distribution $\mathfrak{M}\left(\nu, z_{3}^{2}\right)=\mathcal{M}\left(\nu, z_{3}^{2}\right) / \mathcal{M}\left(0, z_{3}^{2}\right)$.

It can be shown [40] that, for all contributing Feynman diagrams, the Fourier transform of the pseudo-ITD $\mathcal{M}\left(\nu, z_{3}^{2}\right)$ [and, hence, of $\mathfrak{M}\left(\nu, z_{3}^{2}\right)$ ] with respect to $\nu$ has the $0 \leq x \leq 1$ support. In other words, for any $z_{3}^{2}$, we may write

$$
\mathfrak{M}\left(\nu, z_{3}^{2}\right)=\int_{0}^{1} d x e^{i x \nu} \Phi\left(x, z_{3}^{2}\right)
$$

where $\Phi\left(x, z_{3}^{2}\right)$ is the pion pseudodistribution amplitude (pseudo-DA). Sometimes it is convenient to use the $(-z / 2, z / 2)$ endpoints instead of $(0, z)$. Using translation invariance, we get

$$
\begin{aligned}
\left\langle 0\left|\bar{\psi}\left(-z_{3} / 2\right) \ldots \psi\left(z_{3} / 2\right)\right| p\right\rangle & =e^{-i \nu / 2} \mathcal{M}\left(\nu, z_{3}^{2}\right) \\
& \equiv \widetilde{\mathcal{M}}\left(\nu, z_{3}^{2}\right) .
\end{aligned}
$$

To apply the general one-loop formula (2.5), we will need also a parametrization of the $\left\langle 0\left|\bar{\psi}\left(u z_{3}\right) \cdots \psi(\bar{v} z)\right| p\right\rangle$ matrix element. Again, by translation invariance,

$$
\begin{aligned}
\left\langle 0\left|\bar{\psi}\left(u z_{3}\right) \ldots \psi\left(\bar{v} z_{3}\right)\right| p\right\rangle & =e^{i u \nu} \mathcal{M}_{0}[(1-u-v) \nu] \\
& =\int_{0}^{1} d y e^{i y \bar{\nu} \nu+i \bar{y} u \nu} \Phi_{0}(y) .
\end{aligned}
$$

This formula just says that the quark at $\bar{v} z_{3}$ has the $y p_{3}$ momentum, while that at $u z_{3}$ carries $\bar{y} p_{3}$.

\section{B. Structure of contributing terms}

Let us start with the evolution terms in Eq. (2.5), i.e., with those accompanied by $\ln \left(z_{3}^{2}\right)$ in Eq. (2.5). Take first the "vertex" part. In this case, 


$$
\begin{aligned}
& \int_{0}^{1} d u \int_{0}^{1} d v\left\langle 0\left|\bar{\psi}\left(u z_{3}\right) \gamma^{0} \gamma_{5} \psi\left(\bar{v} z_{3}\right)\right| p\right\rangle \\
& \quad \times\left\{\delta(u)\left[\frac{\bar{v}}{\bar{v}}\right]_{+}+\delta(v)\left[\frac{\bar{u}}{u}\right]_{+}\right\} \\
& =\int_{0}^{1} d v\left[\frac{\bar{v}}{v}\right]_{+}\left\langle 0\left|\bar{\psi}(0) \gamma^{0} \gamma_{5} \psi\left(\bar{v} z_{3}\right)\right| p\right\rangle \\
& \quad+\int_{0}^{1} d u\left[\frac{\bar{u}}{u}\right]_{+}\left\langle 0\left|\bar{\psi}\left(u z_{3}\right) \gamma^{0} \gamma_{5} \psi\left(z_{3}\right)\right| p\right\rangle .
\end{aligned}
$$

Using translation invariance, we see that this is equal to

$$
\int_{0}^{1} d w\left[\frac{w}{\bar{w}}\right]_{+} \mathcal{M}(w \nu)\left(1+e^{i \bar{w} \nu}\right)
$$

Transforming to the $\widetilde{\mathcal{M}}$-function using Eq. (3.3) in the form $\mathcal{M}(w \nu)=e^{i w \nu / 2} \widetilde{\mathcal{M}}(w \nu)$, we find that Eq. (3.6) reduces to

$$
e^{i \nu / 2} \int_{0}^{1} d u\left[\frac{2 w}{\bar{w}}\right]_{+} \widetilde{\mathcal{M}}(w \nu) \cos (\bar{w} \nu / 2) .
$$

Take now the "box" part. It is given by

$$
\begin{aligned}
& \int_{0}^{1} d u \int_{0}^{1-u} d v\left\langle 0\left|\bar{\psi}\left(u z_{3}\right) \gamma^{0} \gamma_{5} \psi\left(\bar{v} z_{3}\right)\right| p\right\rangle \\
& =\int_{0}^{1} d u \int_{0}^{1-u} d v e^{i u \nu} \mathcal{M}_{0}((1-u-v) \nu) .
\end{aligned}
$$

Changing $u+v=1-w, u=(1-w) \zeta$, integrating over $\zeta$ and switching to the $\widetilde{\mathcal{M}}$-function gives

$$
e^{i \nu / 2} \int_{0}^{1} d w \frac{\sin (\bar{w} \nu / 2)}{\nu / 2} \widetilde{\mathcal{M}}_{0}(w \nu)
$$

Note that if we would calculate the correction to the function $\widetilde{\mathcal{M}}\left(\nu, z_{3}^{2}\right)=e^{-i \nu / 2} \mathcal{M}\left(\nu, z_{3}^{2}\right)$ rather than to $\mathcal{M}\left(\nu, z_{3}^{2}\right)$, the overall factor of $e^{i \nu / 2}$ in Eqs. (3.7) and (3.9) would be absent.

\section{Matching}

In a similar way, one can derive formulas for other terms from Eq. (2.5). As, a result, we obtain an analog of Eq. (2.9), namely

$$
\begin{aligned}
\widetilde{\mathcal{M}}\left(\nu, z_{3}^{2}\right)= & {\left[1-\frac{\alpha_{s}}{2 \pi} C_{F} Z\left(z_{3}\right)\right] \widetilde{\mathcal{M}}_{0}(\nu) } \\
& -\frac{\alpha_{s}}{2 \pi} C_{F} \int_{0}^{1} d w \widetilde{\mathcal{M}}_{0}(w \nu)\left\{\ln \left[z_{3}^{2} \mu_{\mathrm{IR}}^{2} \frac{e^{2 \gamma_{E}+1}}{4}\right]\right. \\
& \times\left(\left[\frac{2 w}{1-w}\right]_{+} \cos (\bar{w} \nu / 2)+\frac{\sin (\bar{w} \nu / 2)}{\nu / 2}\right) \\
& \left.+4\left[\frac{\ln (1-w)}{1-w}\right]_{+} \cos (\bar{w} \nu / 2)-2 \frac{\sin (\bar{w} \nu / 2)}{\nu / 2}\right\} .
\end{aligned}
$$

To form the reduced pseudo-ITD,

$$
\widetilde{\mathfrak{M}}\left(\nu, z_{3}^{2}\right) \equiv \frac{\widetilde{\mathcal{M}}\left(\nu, z_{3}^{2}\right)}{\widetilde{\mathcal{M}}\left(0, z_{3}^{2}\right)},
$$

we need $\widetilde{\mathcal{M}}\left(0, z_{3}^{2}\right)$, which is given by

$$
\begin{aligned}
\widetilde{\mathcal{M}}\left(0, z_{3}^{2}\right)= & {\left[1-\frac{\alpha_{s}}{2 \pi} C_{F} Z\left(z_{3}\right)\right] \widetilde{\mathcal{M}}_{0}(\nu) } \\
& -\frac{\alpha_{s}}{2 \pi} C_{F} \frac{1}{2} \widetilde{\mathcal{M}}_{0}(0)\left\{\ln \left[z_{3}^{2} \mu_{\mathrm{IR}}^{2} \frac{e^{2 \gamma_{E}+1}}{4}\right]-2\right\} .
\end{aligned}
$$

Thus, the $\sin (\bar{w} \nu / 2) /(\nu / 2)$ terms present in Eq. (3.10) change into $\sin (\bar{w} \nu / 2) /(\nu / 2)-\frac{1}{2} \delta(\bar{w})$ in the expression for the reduced pseudo-ITD. This combination does not have a plus-prescription form, i.e., it differs from $[\sin (\bar{w} \nu / 2) /(\nu / 2)]_{+}$, in contrast to the PDF case, when $(1-w)-\frac{1}{2} \delta(\bar{w})$ could be written as $(1-w)_{+}$.

However, just like in the PDF case, the $Z\left(z_{3}\right)$ term drops from the $\mathcal{O}\left(\alpha_{s}\right)$ correction to the reduced pseudo-ITD. As a result, the matching condition in the pion DA case is

$$
\begin{aligned}
& \tilde{\mathcal{I}}\left(\nu, \mu^{2}\right) \\
& =\widetilde{\mathfrak{M}}\left(\nu, z_{3}^{2}\right)+\frac{\alpha_{s}}{2 \pi} C_{F} \int_{0}^{1} d w \widetilde{\mathfrak{M}}\left(w \nu, z_{3}^{2}\right)\left\{\ln \left[z_{3}^{2} \mu^{2} \frac{e^{2 \gamma_{E}+1}}{4}\right]\right. \\
& \quad \times\left(\left[\frac{2 w}{1-w}\right]_{+} \cos (\bar{w} \nu / 2)+\frac{\sin (\bar{w} \nu / 2)}{\nu / 2}-\frac{1}{2} \delta(\bar{w})\right) \\
& \left.\quad+4\left[\frac{\ln (1-w)}{1-w}\right]_{+} \cos (\bar{w} \nu / 2)-2 \frac{\sin (\bar{w} \nu / 2)}{\nu / 2}+\delta(\bar{w})\right\} .
\end{aligned}
$$

The "tilde" ITD $\tilde{\mathcal{I}}\left(\nu, \mu^{2}\right)$ is related to the light-cone pion DA $\Phi\left(x, \mu^{2}\right)$ by

$$
\tilde{\mathcal{I}}\left(\nu, \mu^{2}\right)=\int_{0}^{1} d x e^{i(x-1 / 2) \nu} \Phi\left(x, \mu^{2}\right) .
$$

Again, the simplest way to extract $\Phi\left(x, \mu^{2}\right)$ is to assume some parametrization for it, like $N(x \bar{x})^{a}$, and fit $a$ from the lattice data on $\tilde{\mathcal{I}}\left(\nu, \mu^{2}\right)$. 
Alternatively, in analogy with Eq. (2.21), one may write $\widetilde{\mathfrak{M}}\left(\nu, z_{3}^{2}\right)$ in terms of $\Phi\left(x, \mu^{2}\right)$ and fit $\alpha_{s}$ and the parameters of the $\Phi\left(x, \mu^{2}\right)$ model using the lattice data for $\widetilde{\mathfrak{M}}\left(\nu, z_{3}^{2}\right)$. The analog of $R\left(x \nu, z_{3}^{2} \mu^{2}\right)$ of Eq. (2.21) is also straightforward-calculable as a closed-form expression.

A few more words about the lattice implementation. While the function $\widetilde{\mathfrak{M}}\left(\nu, z_{3}^{2}\right)$ is directly given by the matrix element of the operator with the $\left(-z_{3} / 2, z_{3} / 2\right)$ endpoints, a more practical way to calculate it is to use the $\left(0, z_{3}\right)$ endpoints and multiply the function $\mathfrak{M}\left(\nu, z_{3}^{2}\right)$ obtained in this way by the $e^{-i \nu / 2}$ factor to get $\widetilde{\mathfrak{M}}\left(\nu, z_{3}^{2}\right)$. The reason is that $z_{3} / 2$ on the lattice should be an integer multiple of the lattice spacing $a$, say $z_{3} / 2=n a$. But then $z_{3}=2 n a$, i.e., the total separations $z_{3}$ given by an odd number of lattice spacings are lost if one uses the $\left(-z_{3} / 2, z_{3} / 2\right)$ endpoints.

\section{Checking the ERBL kernel}

While the matching formula (3.13) has a more involved form than that for PDFs, the difference is basically the presence of sines and cosines of $w \nu / 2$, which are smooth functions of $w$.

On the other hand, it is well known that the ERBL (for Efremov-Radyushkin-Brodsky-Lepage [38,41,42]) kernel $V(x, y)$ governing the evolution of the pion DA is given by different functions for $x<y$ and $x>y$, i.e., it is only piecewise smooth, with singularities like cusps, etc., for $x=y$. So, one may wonder if Eq. (3.10) correctly reproduces the ERBL evolution equation

$$
\delta \Phi\left(x, z_{3}^{2}\right)=-\frac{\alpha_{s}}{2 \pi} C_{F} \ln \left(z_{3}^{2}\right) \int_{0}^{1} d y V(x, y) \Phi_{0}(y)+\cdots .
$$

Let us take first the "vertex" part corresponding to Eq. (3.6) and write it in terms of the DA,

$$
\begin{aligned}
& \int_{0}^{1} d w\left[\frac{w}{\bar{w}}\right]_{+} \mathcal{M}(w \nu)\left(1+e^{i \bar{w} \nu}\right) \\
& =\int_{0}^{1} d w\left[\frac{w}{\bar{w}}\right]_{+} \int_{0}^{1} d y e^{i y w \nu} \Phi_{0}(y)\left(1+e^{i \bar{w} \nu}\right) .
\end{aligned}
$$

Applying the Fourier transformation

$$
\Phi\left(x, z_{3}^{2}\right)=\frac{1}{2 \pi} \int_{-\infty}^{\infty} d \nu e^{-i x \nu} \mathfrak{M}\left(\nu, z_{3}^{2}\right)
$$

that converts $\mathcal{M}(\nu)$ into $\Phi(x)$, gives

$$
\begin{aligned}
& \int_{0}^{1} d w\left[\frac{w}{\bar{w}}\right]_{+}[\delta(x-w y)+\delta(-\bar{x}+w \bar{y})] \\
& =\left[\frac{y / x}{x-y}\right]_{+} \theta(y<x)+\left[\frac{\bar{y} / \bar{x}}{y-x}\right]_{+} \theta(y>x),
\end{aligned}
$$

which is a well-known part of the ERBL kernel $V(x, y)$ (see, e.g., Ref. [38]). As expected, it has different analytic forms in the regions $x<y$ and $x>y$.

For the "box" part given by Eq. (3.8), we have

$$
\begin{aligned}
& \int_{0}^{1} d u \int_{0}^{1-u} d v e^{i u \nu} \mathcal{M}((1-u-v) \nu) \\
& =\int_{0}^{1} d u \int_{0}^{1-u} d v e^{i u \nu} \int_{0}^{1} d y e^{i y(1-u-v) \nu} \Phi(y) .
\end{aligned}
$$

Applying the Fourier transform (3.17) gives the remaining part

$$
\begin{aligned}
& \int_{0}^{1} d u \int_{0}^{1-u} d v \delta(x-y-u+y(u+v)) \\
& =\frac{x}{y} \theta(x \leq y)+\frac{\bar{x}}{\bar{y}} \theta(x \leq y)
\end{aligned}
$$

of the ERBL kernel $V(x, y)$. As a function of $x$, it is given by two straight lines intersecting at $x=y$, with a cusp at this point. Its integral over $x$ gives $1 / 2$, and the $-\frac{1}{2} \delta(1-w)$ term in Eq. (3.13) gives the contribution $-\frac{1}{2} \delta(x-y)$ that provides the plus-prescription for the kernel of Eq. (3.20).

\section{MATCHING FOR GPDS}

\section{A. Definitions and kinematics}

For the pion, one may define the light-cone GPDs $H\left(x, \xi, t ; \mu^{2}\right)$ [43] (see also Refs. [44,45]) at a factorization scale $\mu$ by

$$
\begin{aligned}
& \left\langle p_{2}\left|\bar{\psi}(-z / 2) \gamma^{\alpha} \hat{E}(-z / 2, z / 2 ; A) \psi(z / 2)\right| p_{1}\right\rangle \\
& =2 \mathcal{P}^{\alpha} \int_{-1}^{1} d x e^{-i x(\mathcal{P} z)} H\left(x, \xi, t ; \mu^{2}\right),
\end{aligned}
$$

where $\mathcal{P}=\left(p_{1}+p_{2}\right) / 2$ is the average momentum, the

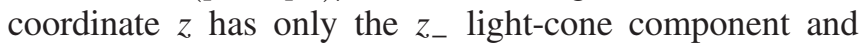
$\alpha=+$. The invariant momentum transfer is given by $t=\left(p_{1}-p_{2}\right)^{2}$. In principle, the r.h.s. of Eq. (4.1) has also the $r^{\alpha}$ term, where $r=p_{1}-p_{2}$ is the momentum transfer. However, the convention is to write $r^{+}=\xi \mathcal{P}^{+}$, where $\xi$ is the skewness variable. In general, $\xi$ may be defined as

$$
\xi=\frac{\left(p_{1} z\right)-\left(p_{2} z\right)}{\left(p_{1} z\right)+\left(p_{2} z\right)} .
$$

For the nucleon, a similar definition holds for the spin nonflip GPD $H\left(x, \xi, t ; z^{2}\right)$, with $2 \mathcal{P}^{+}$substituted by $\bar{u}\left(p_{2}\right) \gamma^{+} u\left(p_{1}\right)$.

On the lattice, as discussed above, it is more convenient to take the $\bar{\psi}(0) \ldots \psi(z)$ operator. By translation invariance, 


$$
\begin{aligned}
& \left\langle p_{2}|\bar{\psi}(0) \ldots \psi(z)| p_{1}\right\rangle \\
& =e^{-i\left(p_{1} z\right) / 2+i\left(p_{2} z\right) / 2}\left\langle p_{2}|\bar{\psi}(-z / 2) \ldots \psi(z / 2)| p_{1}\right\rangle .
\end{aligned}
$$

To introduce pseudo-GPDs, we choose $z=z_{3}$. Then only the value of the third component of the average momentum $\mathcal{P}$ is essential in the scalar product $(\mathcal{P} z)$. So, we can take $p_{1}=\left\{E_{1}, \Delta_{1, \perp}, P_{1}\right\}$ and $p_{2}=\left\{E_{2}, \Delta_{2, \perp}, P_{2}\right\}$. As a result, we have two Ioffe-time invariants $\nu_{1} \equiv-\left(p_{1} z\right)=$ $P_{1} z_{3}$ and $\nu_{2} \equiv-\left(p_{2} z\right)=P_{2} z_{3}$. The skewness variable $\xi$ in this case is given by

$$
\xi=\frac{\left(p_{1} z_{3}\right)-\left(p_{2} z_{3}\right)}{\left(p_{1} z_{3}\right)+\left(p_{2} z_{3}\right)}=\frac{P_{1}-P_{2}}{P_{1}+P_{2}}=\frac{\nu_{1}-\nu_{2}}{\nu_{1}+\nu_{2}} .
$$

Note, that if we choose $\alpha=0$, then both $z^{\alpha}$ and $\Delta_{\perp}^{\alpha}$ contributions will be absent in the parametrization of $\left\langle p_{2}\left|\bar{\psi}(-z / 2) \gamma^{\alpha} \hat{E}(-z / 2, z / 2 ; A) \psi(z / 2)\right| p_{1}\right\rangle$. Hence, we can define the double Ioffe-time pseudodistribution $M\left(\nu_{1}, \nu_{2}, t ; z_{3}^{2}\right)$

$$
\left\langle p_{2}\left|\bar{\psi}(0) \gamma^{0} \ldots \psi\left(z_{3}\right)\right| p_{1}\right\rangle=2 \mathcal{P}^{0} M\left(\nu_{1}, \nu_{2}, t ; z_{3}^{2}\right) .
$$

Using the $\xi$-definition (4.4), we may write $P_{1}=$ $(1+\xi) P$ and $P_{2}=(1-\xi) P$, where $P \equiv \mathcal{P}_{3}$. Denoting

$$
\nu=\frac{\nu_{1}+\nu_{2}}{2},
$$

we define the generalized Ioffe-time pseudodistribution (pseudo-GITD) by

$$
M\left(\nu_{1}, \nu_{2}, t ; z_{3}^{2}\right)=\mathcal{M}\left(\nu, \xi, t ; z_{3}^{2}\right),
$$

and parametrize it by the pseudo-GPD

$$
\mathcal{M}\left(\nu, \xi, t ; z_{3}^{2}\right)=e^{i \xi \nu} \int_{-1}^{1} d x e^{i x \nu} \mathcal{H}\left(x, \xi, t ; z_{3}^{2}\right) .
$$

This formula tells us that the third momentum component of the quark at the point $z_{3}$ is $(x+\xi) P$, as expected. The inverse transformation is given by

$$
\mathcal{H}\left(x, \xi, t ; z_{3}^{2}\right)=\frac{1}{2 \pi} \int_{-\infty}^{\infty} d \nu e^{-i(x+\xi) \nu} \mathcal{M}\left(\nu, \xi, t ; z_{3}^{2}\right) .
$$

Note that originally we had two Ioffe-time parameters $\nu_{1}$ and $\nu_{2}$. However, the Fourier representation (4.9) involves integration over just one $\nu$-parameter, proportional to their sum. The difference $\nu_{1}-\nu_{2}$ is expressed in terms of $\nu$ and the skewness $\xi$ that plays the role of an external fixed parameter like $t$ or $z_{3}^{2}$.

Just like in the pion DA case, it is convenient to introduce the "tilde" pseudo-GITD

$$
\widetilde{\mathcal{M}}\left(\nu, \xi, t ; z_{3}^{2}\right)=e^{-i \xi \nu} \mathcal{M}\left(\nu, \xi, t ; z_{3}^{2}\right)
$$

that is directly conjugate to the pseudo-GPD

$$
\widetilde{\mathcal{M}}\left(\nu, \xi, t ; z_{3}^{2}\right)=\int_{-1}^{1} d x e^{i x \nu} \mathcal{H}\left(x, \xi, t ; z_{3}^{2}\right) .
$$

In deriving the matching relation, we will also need the representation

$$
\begin{aligned}
& \left\langle p_{2}\left|\bar{\psi}\left(u z_{3}\right) \ldots \psi\left(\bar{v} z_{3}\right)\right| p_{1}\right\rangle \\
& \quad=e^{i\left(\nu_{1}-\nu_{2}\right) u} \mathcal{M}_{0}(\nu(1-u-v), \xi) \\
& \quad=e^{i 2 \xi \nu u} \mathcal{M}_{0}(w \nu, \xi) .
\end{aligned}
$$

\section{B. Structure of contributing terms}

Let us now collect the terms resulting from taking Eq. (2.5) between the $\left\langle p_{2}|\ldots| p_{1}\right\rangle$ brackets. Take first the "vertex" part. Proceeding as in the DA case, we start with

$$
\begin{aligned}
& \int_{0}^{1} d v\left[\frac{\bar{v}}{v}\right]_{+} \bar{\psi}(0) \gamma^{\alpha} \psi\left(\bar{v} z_{3}\right) \\
& +\int_{0}^{1} d u\left[\frac{\bar{u}}{u}\right]_{+} \bar{\psi}\left(u z_{3}\right) \gamma^{\alpha} \psi\left(z_{3}\right) .
\end{aligned}
$$

Taking matrix elements we arrive at

$$
\begin{aligned}
& \int_{0}^{1} d v\left[\frac{\bar{v}}{v}\right]_{+} \mathcal{M}_{0}(\nu(1-v), \xi) \\
& +\int_{0}^{1} d u\left[\frac{\bar{u}}{u}\right]_{+} e^{i 2 \xi \nu u} \mathcal{M}_{0}(\nu(1-u), \xi) \\
& =\int_{0}^{1} d w\left[\frac{w}{\bar{w}}\right]_{+}\left(1+e^{i 2 \xi \nu \bar{w}}\right) \mathcal{M}_{0}(w \nu, \xi) .
\end{aligned}
$$

Switching to the $\widetilde{\mathcal{M}}_{0}$-function, we transform this expression into

$$
e^{i \xi \nu} \int_{0}^{1} d w\left[\frac{2 w}{1-w}\right]_{+} \cos (\xi \nu \bar{w}) \widetilde{\mathcal{M}}_{0}(w \nu, \xi) .
$$

Again, the overall $e^{i \xi \nu}$ factor tells us that this is a correction to the $\mathcal{M}$-function written in terms of the $\widetilde{\mathcal{M}}_{0}$-functions.

To check what kind of evolution kernel we have now, write the last line of Eq. (4.14) in terms of the GPD. This gives

$$
\begin{aligned}
\int_{0}^{1} d w\left[\frac{w}{\bar{w}}\right]_{+}\left(1+e^{i 2 \xi \nu \bar{w}}\right) \mathcal{M}_{0}(w \nu, \xi) \\
\quad=\int_{0}^{1} d w\left[\frac{w}{\bar{w}}\right]_{+}\left(1+e^{i 2 \xi \nu \bar{w}}\right) \int_{-1}^{1} d y e^{i(y+\xi) \nu w} H_{0}(y, \xi) .
\end{aligned}
$$

Applying the Fourier transformation (4.9) that converts $\mathcal{M}$ into $H$, we get the following representation for the "vertex" part of the GPD evolution kernel 


$$
\begin{aligned}
K_{v}(x, y ; \xi)= & \int_{0}^{1} d w\left[\frac{w}{\bar{w}}\right]_{+} \int_{-1}^{1} d y[\delta((y+\xi) w-(x+\xi)) \\
& +\delta((y-\xi) w-(x-\xi))] .
\end{aligned}
$$

It is easy to check that, for $\xi=0$, this expression gives the "vertex" part of the AP kernel, while for $\xi=1$ it gives the "vertex" part (3.18) of the ERBL kernel.

Consider now the "box" part. Then we deal with

$$
\int_{0}^{1} d u \int_{0}^{1-u} d v e^{i 2 \xi \nu u} \mathcal{M}_{0}((1-u-v) \nu) .
$$

Changing $u+v=1-w, u=(1-w) \zeta$, integrating over $\zeta$ and switching to the $\widetilde{\mathcal{M}}$-function gives

$$
e^{i \xi \nu} \int_{0}^{1} d w \frac{\sin (\bar{w} \xi \nu)}{\xi \nu} \widetilde{\mathcal{M}}_{0}(w \nu) .
$$

Just like in Eq. (4.15), we have here an overall factor of $e^{i \xi \nu}$, as expected.

Further steps go absolutely in parallel with the derivation of the matching relation for the pion DA. Skipping these steps, we present here the final result

$$
\begin{aligned}
\tilde{\mathcal{I}}\left(\nu, \xi, t, \mu^{2}\right)= & \widetilde{\mathfrak{M}}\left(\nu, \xi, t, z_{3}^{2}\right)+\frac{\alpha_{s}}{2 \pi} C_{F} \int_{0}^{1} d w \widetilde{\mathfrak{M}}\left(w \nu, \xi, t, z_{3}^{2}\right)\left\{\ln \left[z_{3}^{2} \mu^{2} \frac{e^{2 \gamma_{E}+1}}{4}\right]\right. \\
& \times\left(\left[\frac{2 w}{1-w}\right]_{+} \cos (\bar{w} \xi \nu)+\frac{\sin (\bar{w} \xi \nu)}{\xi \nu}-\frac{1}{2} \delta(\bar{w})\right) \\
& \left.+4\left[\frac{\ln (1-w)}{1-w}\right]_{+} \cos (\bar{w} \xi \nu)-2 \frac{\sin (\bar{w} \xi \nu)}{\xi \nu}+\delta(\bar{w})\right\}
\end{aligned}
$$

that gives the light-cone GITD

$$
\tilde{\mathcal{I}}\left(\nu, \xi, t, \mu^{2}\right)=\int_{-1}^{1} d x e^{i x \nu} H\left(x, \xi, t ; \mu^{2}\right)
$$

in terms of the reduced pseudo-GITD

$$
\widetilde{\mathfrak{M}}\left(\nu, \xi, t, z_{3}^{2}\right) \equiv \frac{\widetilde{\mathcal{M}}\left(\nu, \xi, t, z_{3}^{2}\right)}{\widetilde{\mathcal{M}}\left(0,0,0, z_{3}^{2}\right)} .
$$

To extract $H\left(x, \xi, t ; \mu^{2}\right)$, we again propose to take some parametrization for it, and then fit the parameters using the lattice data on $\widetilde{\mathfrak{M}}\left(\nu, \xi, t, z_{3}^{2}\right)$. Doing this, one should keep in mind that the GPD has a nontrivial polynomiality property [43-45]. It amounts to the requirement that, in the nonsinglet case, its $x^{N}$ moment should be a polynomial of the $N$ th degree in $\xi$. A possible way to satisfy it is to use the double distribution Ansatz [46].

An equivalent alternative strategy, similar to that in the PDF and DA cases, is to start with the matching relation between the reduced pseudo-GITD $\widetilde{\mathfrak{M}}\left(\nu, \xi, t, z_{3}^{2}\right)$ and the light-cone GITD $\tilde{\mathcal{I}}\left(\nu, \xi, t, \mu^{2}\right)$ written in terms of $H\left(x, \xi, t ; \mu^{2}\right)$ through Eq. (4.21), and fit the parameters of $H\left(x, \xi, t ; \mu^{2}\right)$ from the lattice data on $\widetilde{\mathfrak{M}}\left(\nu, \xi, t, z_{3}^{2}\right)$.

\section{Remarks on lattice implementation}

Just like in the pion DA case, on the lattice it is more practical to measure matrix elements $M\left(\nu_{1}, \nu_{2}, t ; z_{3}^{2}\right)$ of the operators with the $(0, z)$ endpoints, and then to multiply them by $e^{-i \xi \nu}=e^{-i\left(\nu_{1}-\nu_{2}\right) / 2}$ to convert the result into the
$\widetilde{\mathcal{M}}\left(\nu, \xi, t, z_{3}^{2}\right)$ functions corresponding to the $(-z / 2, z / 2)$ endpoints.

Furthermore, on the lattice, the measurements will be done on a discrete set of values for coordinates $z_{3}=n_{z} a$ and longitudinal momenta $P_{1}=2 \pi N_{1} / L, P_{2}=2 \pi N_{2} / L$, where $L=n a$ is the lattice size in the $z_{3}$ direction. Thus, possible values of the Ioffe-time parameters are limited to discrete sets $\nu_{1}=2 \pi n_{z} N_{1} / n$ and $\nu_{2}=2 \pi n_{z} N_{2} / n$. Correspondingly, possible values for skewness are given by a set of rational numbers

$$
\xi=\frac{P_{1}-P_{2}}{P_{1}+P_{2}}=\frac{N_{1}-N_{2}}{N_{1}+N_{2}} .
$$

In particular, changing $N_{1}$ and $N_{2}$ from 0 to 6 , gives 13 possible values for $\xi$ ranging from 0 to 1 and rather well representing the whole $0 \leq \xi \leq 1$ segment.

However, varying the value of $\xi$ also changes the value of the momentum transfer $t$. Namely, taking purely longitudinal momenta

$$
\begin{aligned}
& p_{1}=\left\{E_{1}, 0_{\perp}, P_{1}\right\}=\left\{E_{1}, 0_{\perp},(1+\xi) P\right\} \\
& p_{2}=\left\{E_{2}, 0_{\perp}, P_{2}\right\}=\left\{E_{2}, 0_{\perp},(1-\xi) P\right\}
\end{aligned}
$$

with

$$
E_{1}=\sqrt{M^{2}+P_{1}^{2}}, \quad E_{2}=\sqrt{M^{2}+P_{2}^{2}},
$$

we get 


$$
\begin{aligned}
t & =-\frac{2 M^{2}\left(P_{1}-P_{2}\right)^{2}}{M^{2}+P_{1} P_{2}+\sqrt{M^{2}+P_{1}^{2}} \sqrt{M^{2}+P_{2}^{2}}} \\
& \equiv t_{0}\left(P_{1}, P_{2}, M\right),
\end{aligned}
$$

or, in the $\{\xi, P\}$ variables,

$$
t_{0}=-\frac{8 \xi^{2} M^{2}}{1-\xi^{2}+\frac{M^{2}}{P^{2}}+\sqrt{\left(1-\xi^{2}+\frac{M^{2}}{P^{2}}\right)^{2}+4 \xi^{2} \frac{M^{2}}{P^{2}}}} .
$$

When $M^{2} / P^{2}$ is small (this is not a very realistic situation for the nucleon, but still), we have

$$
t_{0} \approx-\frac{4 \xi^{2} M^{2}}{1-\xi^{2}}
$$

For small $\xi$, we can approximate

$$
t_{0} \approx-\frac{4 \xi^{2} M^{2}}{1+M^{2} / P^{2}}
$$

In these formulas, $t_{0}$ increases when $\xi$ increases. In any case, this value of $t$ is $\xi$-dependent, while we need to extract GPDs as functions of $x$ for fixed $\xi$ and $t$.

To solve this problem, one may add a transverse component $\Delta_{\perp}$ to the momentum transfer. We propose to use $p_{1}=\left\{E_{1}, \Delta_{\perp}, P_{1}\right\}$ and $p_{2}=\left\{E_{2}, 0_{\perp}, P_{2}\right\}$. Just like in the $z_{3}$ case, the asymmetric choice $\left(\Delta_{\perp}, 0_{\perp}\right)$ on the lattice increases the number of possible discrete values for $\Delta_{\perp}$ compared to the $\left(\Delta_{\perp} / 2,-\Delta_{\perp} / 2\right)$ choice. Then

$$
\begin{aligned}
t= & 2 M^{2}+2 P_{1} P_{2}-\Delta_{\perp}^{2} \\
& -2 \sqrt{M^{2}+P_{1}^{2}+\Delta_{\perp}^{2}} \sqrt{M^{2}+P_{2}^{2}} .
\end{aligned}
$$

Since we will have a discrete set of possible $\Delta_{\perp}^{2}$ values on the lattice, it is impossible to arrange exactly the same value of $t$ for different values of $\xi$. A more modest goal is to collect a set of data with close values of $t$, and then make interpolation to a chosen $t$-value.

Another strategy is to choose first some particular values of $P_{1}$ and $P_{2}$. This fixes the value of $\xi$. The next step is to take several different values of $\Delta_{\perp}$ to change $t$. That will give the $t$-dependence for fixed $\xi$ and $\nu$. After this, changing $z_{3}$, we will change $\nu$ leaving $\xi$ and $t$ unchanged. Finally, using the matching conditions to convert the $\nu$-dependence into the $x$-dependence, we will end up with $H\left(x, \xi, t ; \mu^{2}\right)$ for a fixed $\xi$ as a function of $x$ and $t$.

\section{SUMMARY}

In this paper, we have derived the matching relations for the pion distribution amplitude and nonsinglet generalized parton distributions that connect them with their off-thelight-cone counterparts, the pseudo-DA and pseudo-GPDs. The latter may be calculated in lattice simulations, and the matching relations are crucial in converting them into the experimentally measurable (in principle) light-cone parton distributions.

We have also derived matching relations for the usual parton distribution functions. One of them, given by Eq. (2.22), allows to express the lattice-measurable reduced pseudo-ITD $\mathfrak{M}\left(\nu, z_{3}^{2}\right)$ with its $\operatorname{PDF} f\left(x, \mu^{2}\right)$. Similar relations may be derived for the lattice matrix elements renormalized using the RI/MOM schemes. Then one may be able to directly fit these matrix elements by a chosen model for the PDF.

The main feature of our derivations is that we start with a universal expression for the one-loop correction in an operator form. In particular, we show how this universal expression produces particular matching conditions for ITDs related to different parton distributions. In fact, these different matching relations have a rather similar structure. Also, these relations are much simpler than the matching relations for quasi-PDFs, quasi-DAs and quasi-GPDs given in Refs. [2,11-15].

The matching relations for the pseudo-PDFs have been already used in lattice calculations [10,21,47-49], while these for the pion DA and GPDs will be used in the ongoing lattice calculations.

\section{ACKNOWLEDGMENTS}

I thank K. Orginos and R. Edwards for their interest in this investigation and discussions. This work is supported by Jefferson Science Associates, LLC under U.S. DOE Contract No. DE-AC05-06OR23177 and by U.S. DOE Grant No. DE-FG02-97ER41028.
[1] K. Cichy and M. Constantinou, Adv. High Energy Phys. 2019, 1 (2019).

[2] X. Ji, Phys. Rev. Lett. 110, 262002 (2013).

[3] X. Ji, Sci. China Phys. Mech. Astron. 57, 1407 (2014).
[4] W. Detmold and C. J. D. Lin, Phys. Rev. D 73, 014501 (2006).

[5] V. Braun and D. Müller, Eur. Phys. J. C 55, 349 (2008).

[6] Y. Q. Ma and J. W. Qiu, Phys. Rev. D 98, 074021 (2018). 
[7] Y. Q. Ma and J. W. Qiu, Phys. Rev. Lett. 120, 022003 (2018).

[8] A. V. Radyushkin, Phys. Rev. D 96, 034025 (2017).

[9] A. Radyushkin, Proc. Sci., QCDEV2017 (2017) 021 [arXiv:1711.06031].

[10] K. Orginos, A. Radyushkin, J. Karpie, and S. Zafeiropoulos, Phys. Rev. D 96, 094503 (2017).

[11] X. Xiong, X. Ji, J. H. Zhang, and Y. Zhao, Phys. Rev. D 90, 014051 (2014).

[12] X. Ji and J. H. Zhang, Phys. Rev. D 92, 034006 (2015).

[13] T. Izubuchi, X. Ji, L. Jin, I. W. Stewart, and Y. Zhao, Phys. Rev. D 98, 056004 (2018).

[14] W. Wang and S. Zhao, J. High Energy Phys. 05 (2018) 142.

[15] W. Wang, J. H. Zhang, S. Zhao, and R. Zhu, Phys. Rev. D 100, 074509 (2019).

[16] X. Ji, A. Schäfer, X. Xiong, and J. H. Zhang, Phys. Rev. D 92, 014039 (2015).

[17] X. Xiong and J.H. Zhang, Phys. Rev. D 92, 054037 (2015).

[18] Y. S. Liu, W. Wang, J. Xu, Q. A. Zhang, J. H. Zhang, S. Zhao, and Y. Zhao, Phys. Rev. D 100, 034006 (2019).

[19] X. Ji, J. H. Zhang, and Y. Zhao, Nucl. Phys. B924, 366 (2017).

[20] A. V. Radyushkin, Phys. Lett. B 781, 433 (2018).

[21] A. Radyushkin, Phys. Rev. D 98, 014019 (2018).

[22] J. H. Zhang, J. W. Chen, and C. Monahan, Phys. Rev. D 97, 074508 (2018).

[23] I. I. Balitsky and V. M. Braun, Nucl. Phys. B311, 541 (1989).

[24] B. L. Ioffe, Phys. Lett. 30B, 123 (1969).

[25] A. Radyushkin, Phys. Lett. B 767, 314 (2017).

[26] G. Altarelli and G. Parisi, Nucl. Phys. B126, 298 (1977).

[27] V. N. Gribov and L. N. Lipatov, Yad. Fiz. 15, 781 (1972) [Sov. J. Nucl. Phys. 15, 438 (1972)].

[28] Y. L. Dokshitzer, Zh. Eksp. Teor. Fiz. 73, 1216 (1977) [Sov. Phys. JETP 46, 641 (1977)].
[29] X. Ji, J. H. Zhang, and Y. Zhao, Phys. Rev. Lett. 120, 112001 (2018).

[30] T. Ishikawa, Y. Q. Ma, J. W. Qiu, and S. Yoshida, Phys. Rev. D 96, 094019 (2017).

[31] J. Green, K. Jansen, and F. Steffens, Phys. Rev. Lett. 121, 022004 (2018).

[32] V. Braun, P. Gornicki, and L. Mankiewicz, Phys. Rev. D 51, 6036 (1995).

[33] J. Karpie, K. Orginos, A. Rothkopf, and S. Zafeiropoulos, J. High Energy Phys. 04 (2019) 057.

[34] A. Accardi, L. T. Brady, W. Melnitchouk, J. F. Owens, and N. Sato, Phys. Rev. D 93, 114017 (2016).

[35] K. Cichy, L. Del Debbio, and T. Giani, J. High Energy Phys. 10 (2019) 137.

[36] A. V. Radyushkin, Phys. Lett. B 788, 380 (2019).

[37] A. V. Radyushkin, arXiv:hep-ph/0410276.

[38] G. P. Lepage and S. J. Brodsky, Phys. Rev. D 22, 2157 (1980).

[39] A. V. Radyushkin, Phys. Rev. D 95, 056020 (2017).

[40] A. V. Radyushkin, Phys. Lett. 131B, 179 (1983).

[41] A. V. Efremov and A. V. Radyushkin, Teor. Mat. Fiz. 42, 147 (1980) [Theor. Math. Phys. 42, 97 (1980)]

[42] A. V. Efremov and A. V. Radyushkin, Phys. Lett. 94B, 245 (1980).

[43] X. D. Ji, Phys. Rev. Lett. 78, 610 (1997).

[44] D. Müller, D. Robaschik, B. Geyer, F.-M. Dittes, and J. Hořejši, Fortsch. Phys. 42, 101 (1994).

[45] A. V. Radyushkin, Phys. Rev. D 56, 5524 (1997).

[46] A. V. Radyushkin, Phys. Rev. D 59, 014030 (1998).

[47] J. Karpie, K. Orginos, and S. Zafeiropoulos, J. High Energy Phys. 11 (2018) 178.

[48] B. Joó, J. Karpie, K. Orginos, A. Radyushkin, D. Richards, and S. Zafeiropoulos, arXiv:1908.09771.

[49] B. Joó, J. Karpie, K. Orginos, A. V. Radyushkin, D. G. Richards, R.S. Sufian, and S. Zafeiropoulos, arXiv: 1909.08517 [Phys. Rev. D (to be published)]. 